https://helda.helsinki.fi

\title{
Protein nanotechnology
}

\section{Yaradoddi, Jayachandra}

Springer Nature Switzerland

2019

Yaradoddi , J , Kontro , M H , Ganachari , S V , Sulochana , M B \& Dayanand , A 2019 , Protein nanotechnology . in L Martínez, O Kharissova \& B Kharisov (eds), Handbook of

Ecomaterials : Protein nanotechnology . Springer Nature Switzerland , pp. 3573-3585 . https://doi.org/10.1007/978-3

http://hdl.handle.net/10138/326419

https://doi.org/10.1007/978-3-319-68255-6_192

unspecified

acceptedVersion

Downloaded from Helda, University of Helsinki institutional repository.

This is an electronic reprint of the original article.

This reprint may differ from the original in pagination and typographic detail.

Please cite the original version. 


\title{
PROTEIN NANOTECHNOLOGY
}

Jayachandra S. Yaradoddi ${ }^{1,2,6^{*}}$, Merja Kontro ${ }^{2}$, Sharanabasava V. Ganachari ${ }^{1}$, Sulochana M. B. ${ }^{3}$ Dayanand Agsar ${ }^{4}$, Rakesh Tapaskar ${ }^{5}$ and Ashok S. Shettar ${ }^{1}$

${ }^{1,2}$ Centre for Material Science, Advanced research in Nanoscience \& Nanotechnology, KLE Technological University, Hubballi

and Dept. of Environmental Sciences, University of Helsinki, Finland,

${ }^{3}$ Department of PG Studies and Research in Biotechnology, Gulbarga University, Kalaburagi-585106, INDIA

${ }^{4}$ Department of PG Studies and Research in Microbiology, Gulbarga University, Kalaburagi-585106, INDIA

${ }^{5}$ School of Mechanical Engineering KLE Technological University, Hubballi-580031, INDIA

${ }^{6}$ Extremz Biosciences private limited, CTIE start up street, KLE Technology University campus, Hubballi-580031

\begin{abstract}
:
Medical management is to be preserved well, especially diagnosis is fast, easy and cheap. Sometimes RNA and DNA nanobio-based diagnostic may not give the precise data in respect to specific disorders. Therefore, some quantifiable protein information and molecular folding are very much required for the analysis of such disorders. Proteins in minute concentration is typically not detectable under the normal circumstances. Nowadays which can be measured and quantified using protein nanotechnology methods. On the other side protein machineries brings out the tasks unsafe to cell behavior, comprises DNA duplication, intracellular carriage, ion pump, cellular motility. They have changed unbelievable multiplicity, precision, efficacy and a substantial quantity of study in contemporary biology intended to expose the vital mechanisms or processes primarily their function. This chapter also emphasizes the recent development in protein nanotechnology with special focus on molecular cytoskeletal motors, dyneins, myosin's and kinesins. They constitute subcategory of the protein machineries, they have distinguished properties and can be able to convert biochemical energy directly to automatic work.
\end{abstract}




\section{Introduction:}

Nanotechnology research develops objects ranging between 1-100 nm in size. The Greek term nano means dwarf, and it is $10^{-9} \mathrm{~nm}$. Nanotechnology, denotes the tools and techniques used for understanding, modeling, and manufacturing of materials at the nanometer dimensions. The earliest perception of exploring constituents and living systems on the nanoscale occurred dates back 60 years, scientist Richard Feynman delivered speech in annual American Physical Society meet held at the California Institute of Technology in 1959. His talk on "There is plenty of room at the bottom" [1], is most commonly evaluated as the primary expression within the biosphere of constituents, species, and assemblies in nanoscale levels.

Towards the end of the $20^{\text {th }}$ century, it was observed that certain protein or peptide chains, i. e. protein transduction domains like TAT protein, can directly penetrate into the cell cytoplasm and further to cell nucleus [2,3]. Such nanotechnology methods can be employed in the drug delivery of proteins and other peptides into the cell as drugs. Moreover, the system can be utilized to transport inside cell many other small drug molecules, and larger molecules such as DNA, enzymes and other nanoparticulates, including quantum dots, liposomes and $\mathrm{Fe}^{3+}$ oxide ions [4]. The exact mechanism of the whole processes presently is to be subjected for investigation, even though significant development has been done so far. Some of the data has indicated that the complex electrostatic interactions and the hydrogen bonds of proteins and peptide facilitating the direct transfer of these tiny molecules to the cell $[5,6]$. Foremost important accomplishments in the field of drug distribution was the fabrication of smart drug delivery systems (SDDSs), similarly named as stimuli-susceptible transfer systems. The idea is mainly based on the quick induced conversions in the physico chemical properties of polymeric structures. This inducement stimulus can be physical (mechanical, electrical, temperature, light, ultrasound,), chemical (pH, ionic strength) or biological (biomolecules, enzymes). It may come from inside the cell as a consequential deviation in the physiology of a living organisms, or an external, artificial signal may trigger the biochemical reactions. SDDS elaborately controls drug release in response to numerous cellular stimuli [7].

Nanoparticles as drug carriers have enormous opportunities in living organisms, and in the therapeutic and medical treatments. They are extensively applied in delivering drugs, peptides, proteins, DNA, RNA, vaccines, etc. [8]. Currently, the nanoparticle investigation on drug releasing 
systems is particularly focusing on: (1) the selectivity and controlled drug delivery rate: (2) the surface structures of nanoparticles to improve their specific targeting in an organisms; (3) the optimized synthesis of nanoparticles with the efficient drug transport capacity, and suitability for medicine in various scales; and (4) the in vivo research on active processes, in which nanoparticles are interacting with plasma, cells, tissues or organs, etc.

An unique kind construction, a solid nanoparticle (SLN), or a suspension with compressed fat globules having a solid core at the room temperature can behave as a carrier of molecules. The development of SLN's begins with the liquefying of the solid lipid e.g. by increasing the temperature, followed by combining with water to form an oil in water suspension, and then lipid nanoparticles are solidified by reducing the temperature. Similarly, proteins can be used as a nano transporters. Virus and non-virus proteins are used as a vector to carry DNA into cells, as the pure DNA in a tissue will be fast enzymatically degraded. Moreover, the negatively charged DNA cannot penetrate the cell due to the repulsion by the cell membrane.

Other examples of nanocarrier, includes the avidin-linked gelatin nanoparticles for the delivery of biotinylated peptide nucleic acids (PNA) to examine their usage in antisense treatments [9]. According to Zwiorek and coworkers [10] non-toxic gelatin nanoparticles could be effective in virus free gene transfer. Two-stage disolvation procedure enabled the preparation of homogenous spherical colloids with the cationized surface charge and low polydispersity, though other possible methods for gelatin nanoparticles synthesis occur [11]. Compared with the polyethyleneimineDNA complexes, the gelatin particles with amino acid side chains available for multiple modifications offer possibilities for use in many unique application, such as specific uptake of drugs in the diseased tissue [10]. Transfection through the assistance of positively charged gelatin nanoparticles was also used by Xu and his colleagues [11] for the transfer DNA plasmids encoding for insulin growth factor [IGF-1] into chondrocytes. To integrate negatively charged plasmids with the positively charged gelatin nanoparticles, complex electro statistically-driven separation was found to be feasible, fast and predominantly beneficial method for the integration of large molecules. Circular, positively charged gelatin units linked to plasmids were smaller in size than the corresponding noncationized microparticles. Fluorescence microscopy confirmed that the cationized gelatin nanoparticles were effectively transformed, and IGF-1 expression raised fivefold in the target cells, in contrast to noncationized gelatin particles. Transfection occurred due to 
increased endocytosis as consequences of interactions between the positive charge of the gelatin nanoparticle surface and the negative charge on the cell membrane.

The over expression of IGF-1 in collagen (type II) -glycosaminoglycan scaffolds 3-D cultures was continued for 14 days. IGF-1 has also been recognized to induce growth in cartilage, skeletal muscle and in bones and many other cells/tissues in the body tissues. This method has wide variety of potential applications in gene therapy and tissue manipulations/engineering.

\section{Significance of Protein Nanotechnology}

Cells, organelles, and countless catabolic and anabolic biochemical reactions inside the cell can be considered as an organization of nanoscale devices. Nanotechnology differs from chemistry in an actual basic aspect. While chemistry considers atoms, ions and molecules at the element level, nanotechnology pursues essentially to manipulate distinct particles and atoms in a very precise path. Proteins belong to main cellular molecules that have the key role in maintaining the functionality of the cell. They can perform numbers of functions inside the cell as enzymes that is they are the actuating force for various biological and chemical reactions under cellular conditions. Further, proteins may serve as antibodies that identify foreign particles and permit the immunological system to counteract and remove undesirable agents. As disorders, their treatment, and medications may alter the cellular protein profile, proteomics, or protein profile determination and comparison between healthy and sick people enables to understand disease-related disorders in the metabolism, and the required treatment. Therefore, the knowledge of molecular structures, metabolic activities, and molecular and nanoscale function of proteins is utmost important to understand for regulating living processes by nanoscale drugs. Such information will donate us capability to engineer the molecules of organisms, to increase energy production by microbial or enzymatic biofuel cells, or to sense the human health efficiently analyze and eventually prevent the disease.

Genes determine the amino acid sequence of the proteins. The genetic code of DNA transcribed to the corresponding mRNA sequence, which is then read in the ribosome as the amino acid sequence of proteins. Therefore, the explicit three-dimensional structure of proteins is a result of amino acid chain folding, which is intended to achieve a specific function in a living organism. Proteins are inactive until they have folded correctly into the active form after possible enzymatic cleavages in the post-translational modifications. Moreover, the protein molecules called 
chaperons are extremely important for the correct folding of a large number of proteins to their ultimate form and function inside the cell. A genetic factor can also be involved in diverse splicing mechanisms of proteins. As a result of the post translational alterations, a protein molecule can pass through several intense rearrangements. Accordingly, the gene sequences information of proteins is not adequate to designate all aspects of life. The regulation of the ultimate activities of proteins in their cellular metabolic pathways is also critical, as they are the definitely the most important molecules in the life cycle.

\section{Structure of Protein}

The proteins are elongated chains of 20 most common L-amino acids, the sequence of which determines the primary structure of proteins (Nelson and Cox, 2017). In the protein backbone, the peptide bond is formed between carboxyl group of one amino acid and amino group of another amino acid, simultaneously water molecule is cleaved. Generally, the amino acid chain length of polypeptides is less than 100 amino acids, and the chain length of proteins is typically more than 100 amino acids. The typical molecular weight (MW) of a protein is approximately 15000-70000. One of the largest known protein molecule is human titin, which consists of almost 27000 amino acid residues. The amino acid side chains can be nonpolar aliphatic or aromatic; or polar uncharged, positively charged, or negatively charged. The side chains of amino acids are important for protein folding to develop as barely ordered, 3-dimensional assembly, known as secondary structure. The assembly is then categorized by a low-energy conformation, in which hydrophilic groups are located on surface of the protein molecule, and the hydrophobic groups are inside. The most common secondary structures of proteins are $\alpha$-helix configuration and $\beta$-pleated sheet. These two secondary configurations are among the most important building blocks of the protein structure to form the ultimate tertiary structure, which is stabilized via covalent modifications (e.g. disulfide cross linkages), and weak interactions, such as hydrogen bonding, van der Waals forces, and hydrophilic and hydrophobic interactions. Thus, the tertiary structure is the comprehensive 3D assembly among an undividable protein component. Several protein molecules can be bound together to form a large protein molecule complex called as quaternary structure, which is the maximum level in the protein structure hierarchy. Separate protein molecules are bound together by weak, noncovalent interactions, which allow flexibility in the structure called allosteric 


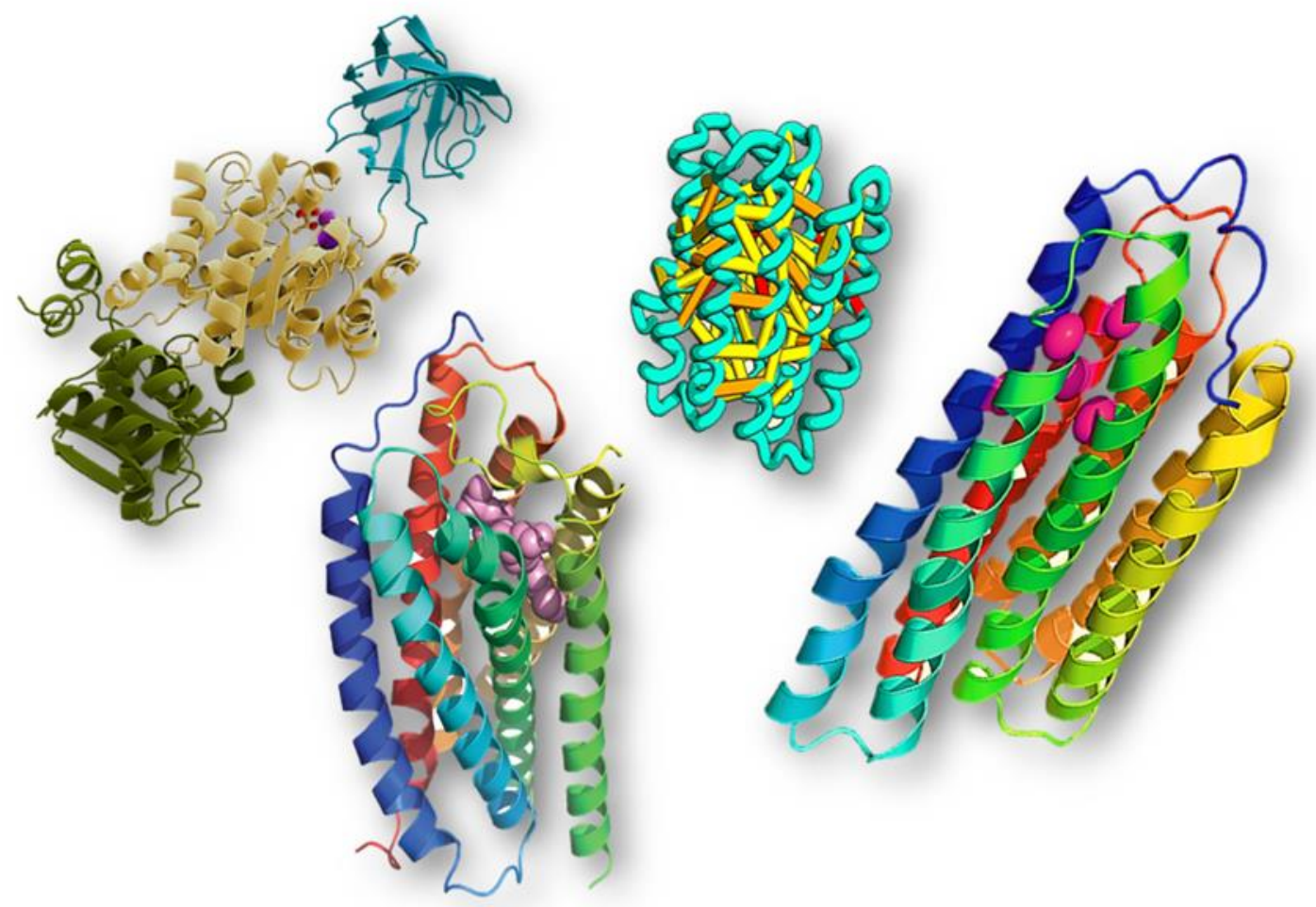

3-D structures of the proteins

Figure 1: Showing 3-dimensional structures of the protein molecule

regulation of protein activity. The 3-dimensional assembly (Figure 1) of proteins is vital in understanding their role. The primary structure offers only a small amount information about the protein function. To determine the functions of proteins, the active form of the three-dimensional, folded protein is required. The structure of the crystallized protein can be determined by X-ray crystallography. Once the structure of an active folded protein is unfolded for example by heating, the protein can undergo a permanent denaturation, such as egg albumin becomes white when heated. After the heat denaturation albumin cannot be reversed to the active form, opposite to some other proteins which can be renatured to their original structure. Many diseases have implications for the problems in protein misfolding, corresponding to mad cow disease, cystic fibrosis, Alzheimer's disease, a congenital for of emphysema, and many tumors. A lot of efforts have been made in theoretical investigations to regulate protein structures. 
Through combination of advanced methods such as nuclear magnetic resonance and mass spectroscopy researchers have identified optimum group of small molecules in production of bidentate solutions for clostridial neurotoxin infections mainly caused by tetanus and botulinum species.

\section{Composite interaction between filaments and motor proteins for the artificial surfaces}

Recurrent practical obstacles in assimilating functional proteins to nanodevices are related to the difficulties in combining artificial constituents with bioorganic molecules without disturbing the protein activity. It is tricky to understand and regulate the interactions between proteins and biomaterials, the problem that has gained much attention [12, 13]. For example, in circumstance of implant biomaterial the principal methods expanding biocompatibility focus on the development of surfaces that do not adsorb proteins, or on the manufacturing surfaces that can absorb proteins without affecting functionality. If these reactions cannot be controlled, implant materials can activate the immune system against them. Because of this important issue, efforts are constantly exerted on protein behavior on surfaces. Still much improvements are required for obtaining accurate results in generating surface markers that tend to attach proteins without affecting their function, and in producing surfaces that entirely tolerate the protein adsorption. Towards

harnessing motor proteins and their movements in cytoskeletal paths can be used in protein nanotechnology, demonstrating their effectiveness in transportation action, it is very vital to regulate the precise behavior of the proteins in surface-related phenomena.

\subsection{Adsorption of Biomolecular Motor}

The finding of kinesin motor action was an outcome of kinesin protein capacity to bind to the surface of the glass and continue its function. When the degradation or denaturation of kinesin could be prevented by the passivation treatment of the surface, kinesin was found to bind to numerous different glass and hydrophilic surfaces [14-17]. One of the best passivation treatment was that done with the milk protein, casein. Casein produces diverse aggregates with diameters ranging between 10-300 $\mathrm{nm}$ diameter in clarification [18]. The degree of casein aggregation varies depending on factors like the calcium ion concentration, degree of phosphorylation, and $\mathrm{pH}$ [19]. The quality of casein is the best for kinesin experiments after solubilization in buffer and filtration to eliminate aggregates [20]. Casein protein significantly rises the motion of kinesin motors adsorbed to the surface; however, mechanisms causing the increased activity are not well 
understood [20]. Employed prototype is like casein aggregates $10 \mathrm{~s} \mathrm{~nm}$ in diameter pack upon surface, end areas of kinesin motors adhere amongst casein units, later motor heads stick inside the solution to interact with microtubules. That is non-excluded with kinesin motors stick straight towards the surface-adsorbed casein, nonetheless excessive casein in the motor suspension does not contest per the surface-adsorbed casein. Similarly, it was stated that casein has a chaperone kind of role to steady these proteins against any sort of denaturation process [21]. We cannot rule out the portion improvement of kinesin function by casein, since steadying the motor protein assembly. Motors stacked path has been designated totally useful, further, the bond is strong sufficient which cannot be removed by the motor activities [22-24]. Although different hydrophilic surfaces support the kinesin-mediated microtubule actions, whereas water hating surfaces does not. Once glass exteriors are treated through hydrophobic surface treatments preceding with the kinesin and casein conduct, no microtubule motion then observed [25, 26]. With this explanation motors losses their original structure on these waters hating surfaces, pretreatment with casein couldn't stops motor denaturation. The indicated stuff of surface chemistry reliant on motor role can be useful in describing, where the transportation of micro fabricated surfaces microtubule takes place.

\subsection{Immobilization of Microtubule}

In different functions somewhere, microtubules are entrapped upon a surface, load functioned kinesins transfer alongside of threads, numerous approaches have been established for immobilization of microtubules. Microtubules will attach to a glass [27], this entrapment will be sufficient for some investigations on motor role. Though, transportation applications, stronger entrapment is normally essential. The most common entrapment processes remain functionalized on glass surfaces through an amino silane complex, it would deliberate a positive charge on the surface $[28,29]$. Microtubule remains negative charge at bodily $\mathrm{pH}$, attach firmly to the surfaces proceeding in higher microtubule concentrations. Through lithographical modelling silane on silicon wafers, this method can be utilized in designing entrapped microtubules at micro measures magnitudes [30]. Poly-lysine treated glass found to be an alternative method and practices electrostatic interactions in binding microtubules to surfaces [31]. 2 additionally microtubule entrapment approaches indicated near exertion well. Microtubules then be biotinylated bind to surfaces by streptavidin, through adsorbing streptavidin straight to the surface, entrapping another 
biotinylated protein and later by means of streptavidin as an adhesive amongst protein molecule besides biotinylated microtubule [32]. Actual sense, self-constructed closely packed molecules a part of the assemblies are finished per biotin can be marked, microtubules and streptavidin marked through primary biotin. Supplementary practices of modelling biotin or streptavidin in nano/ micron scale sizes established for nanoscaled 2D protein designs can be useful in modelling biotinylating microtubules. Ultimate entrapment approach, by means of non-functionalized kinesin motors to entrap the microtubules [33, 34]. Although maximum entrapment method relies on the explicit application, even though nearly universal deliberations adapted to almost wholly microtubules entrapment methods (Figure 2).

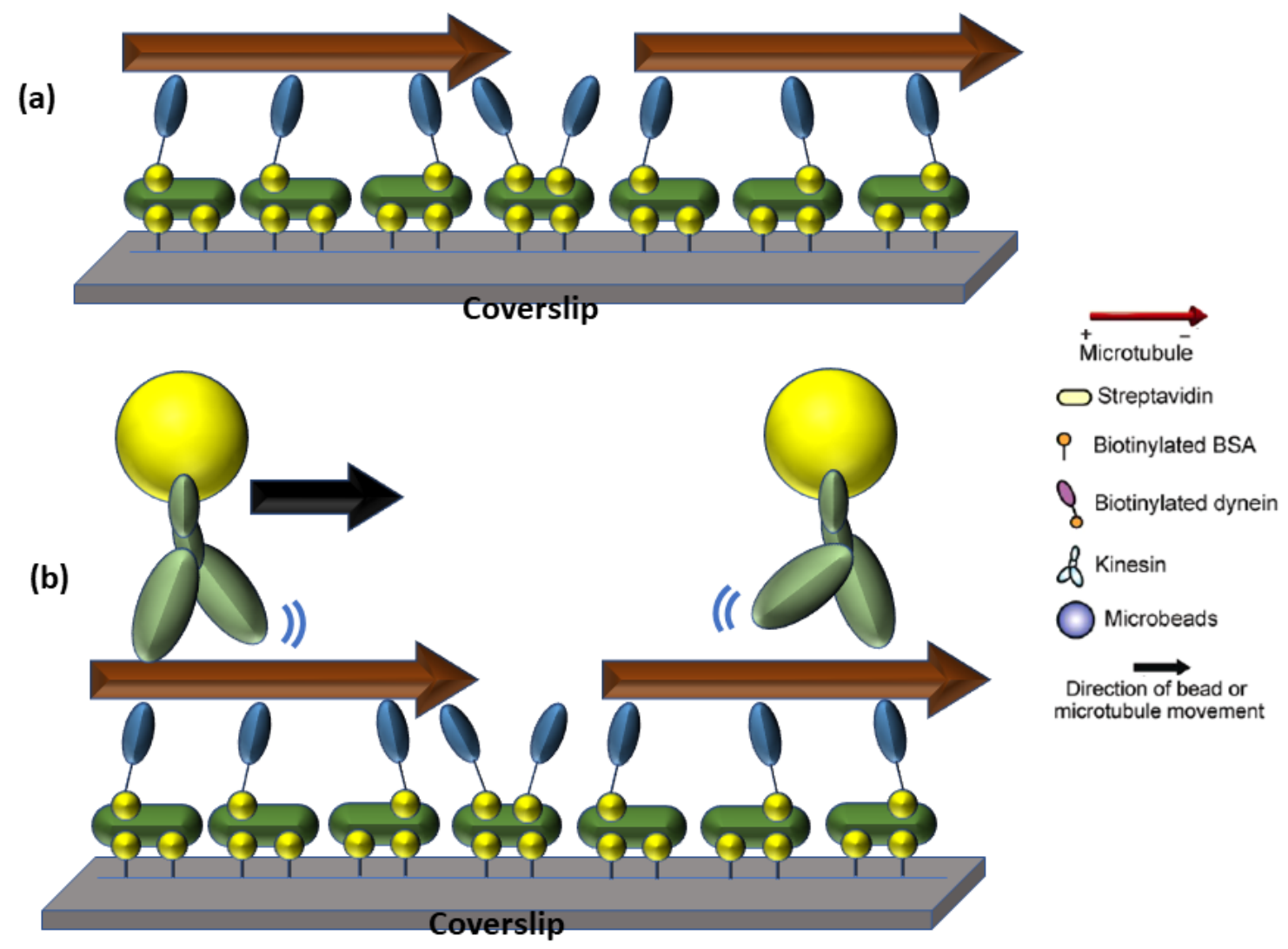

Schematic diagram of Molecular motors

Figure 2: Indicating the complex interaction molecular motors

Firstly, methods permit the modelling microtubules in micron size are useful, if thread direction to be regulated. For example, through thin tiles glue, microtubules can merely lay across the lines, opposing design. 


\subsection{Deliberation for System Design}

Progress in bio motor-mediated micro or nanoscale devices have proceeded into 3 stages: 1) repeating intracellular mobility while under laboratory trials, 2) regulation of microtubule carriage by means of surface processing, micro fabrication, additional useful procedures, and c) generating combined practical devices. Today investigators are revolving to the superior stage in nanodevice development. Still numerous problems needs to be addressed to move the research forward to a more advanced level, for example, more developed protein engineering results, their introduction, the recognition by proteins, and metabolic processing. The development in these guidelines to date, upcoming efforts in these areas are revised here.

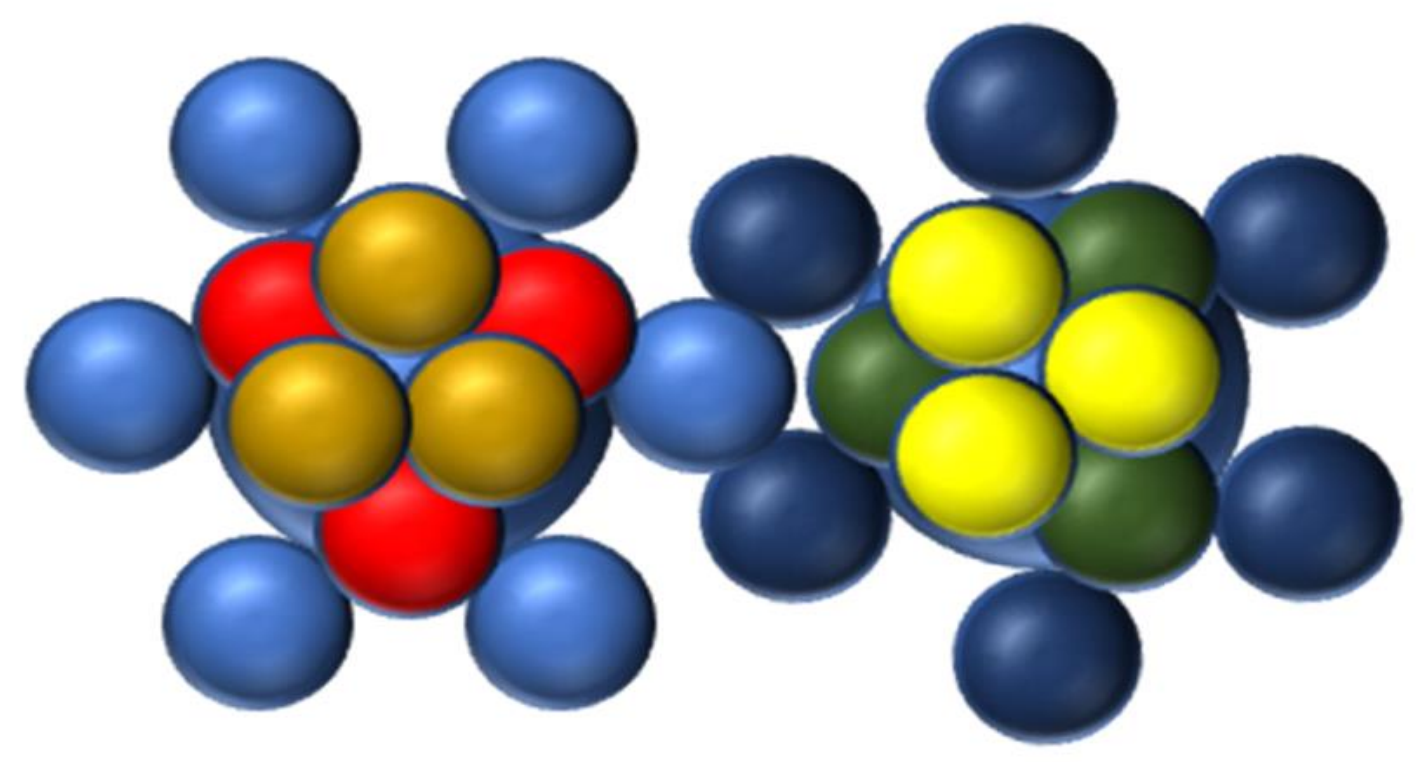

Animation of Molecular motor

Figure 3: Depicting the animated design of molecular motor cells.

\subsection{Increasing the half-life of proteins}

Overall, proteins are much more delicate than other nanoscale materials. For assessing their possible usefulness when combined into diagnostic devices, in order examine substitute design 
methods, there is a requirement to govern duration of microtubules and kinesin motors within usual functioning circumstances, prior in search of sceneries that can prolong their shelf life. Under usual workroom situations, the microtubules that are stabilized by taxol, steady at normal temperature even up to seven days [35,36], the kinesin motor steady for few hrs.-days depends on their quantity or concentrations, temperature, buffer and concerned parameters.

Microscopic small tubules can be imagined by Differential interference contrast (DIC) microscopy, but then application, somewhere motor detected fluorescence is ample useful. Inside the microfluidic area, typical method in generating networks is PDMS molding and attachment to glass [37], in perfect sense these 2 methods can be united. Though, many researchers reviewing microtubule fluorescent activities under the PDMS networks rapidly contended to trouble through photodamage [38]. Within typical microtubule sliding examine, cocktail of microtubule comprises buffer salt, adenosine tri phosphate to provide molecular motion, antifade containing an oxygen molecule scavenging structure also reducing agents. In antifade absence, contact of these luminous microtubules towards higher power mercury lamp applied for fluorescence excitation resulted in fast photobleaching, molecular motion loss, microtubules polymerization since the oxygen free radical damage. By antifade, motion of microtubules which are labeled with rhodamine perceived few minutes under frequent higher power brightness. Deprived of light, microtubule motion persists for about 10 hours. Though, the movement of cell is surrounded by PDMS as an alternative to glass materials, brightness proceeding hindrance in molecular motion and rupture microtubules for few seconds [39]. This source of failure outlined with the higher oxygen penetrability of silicone polymer and overcomes antifade's capacity in order to eliminate overall soluble oxygen.

\subsection{Sample injection and analyte detection}

There are 2 key featured difficulties in generating kinesin-driven systematic nanodevices, they are sample introduction and analyte detection. Till today limited accomplishment has been reported in current field, since the necessity is widespread to nearly all nanosensor systems, thoughts, designs can be undoubtedly taken from other relevant and integrated fields. Concerning to sample addition, suffering from one main limitation is the bio motors works only in liquid phase. However, described above, kinesins could be viable, but for their appropriate role must continue in suitable buffers. Henceforth, to sense floating particles (for example microbes), the samples are required to be intense, later transported to the aqueous phase. Near, obviously numerous manufacturing 
issues to be addressed for specific applications and reliability. The position where the strengths of kinesin-driven approaches optimized is recognition of proteins, RNA molecules or other tiny particles in miniaturing liquid sample. In addition to above, both visual and electrical techniques have been established to fast lysis cells, allowing their intra cellular constituents to be brought to the microanalysis machines [42, 43].

\section{Conclusion}

This chapter envisioned at the current advancements in protein nanotechnologies such as biosensors, protein microarrays, etc., their application in finding out diseases at proteomics level have also been deliberated. Since, the extremely advanced properties of protein machineries, provides the unbelievable potential in assimilating them into hybrid or artificial nanodevices. With their micro or nano scale size contest fine and is parallel to developing nanomanufacturing competences and new useful nanoscaled materials production. An enduring challenge in emerging nanotechnology is to find innovative methods to engineer and establish atoms and also nanoparticles to bag wherever self-structure do not actively promising. Since, the biomolecular motors have changed to transportation and establish biomaterials on the nano and microscale within the cells, they have the conceivably to resolve numerous general issues in protein nanotechnology. The present chapter has also revealed the transportation capacity of kinesin motors with the laboratory conditions and stress towards making microfluidic devices for analyte detection.

\section{References}

[1] Feynman, R. (1960) There's plenty of room at the bottom: an invitation to enter a new field of physics. Eng. Sci. February Issue

[2] Wattiaux, R., Laurent, N., Wattiaux-De Coninck, S., and Jadot, M. (2000) Endosomes, lysosomes: their implication in gene transfer. AdV. Drug DeliVery ReV.41, 201-208.

[3] Caron, N. J., Torrente, Y., Camirand, G., Bujold, M., Chapdelaine, P., Leriche, K., Bresolin, N., and Tremblay, J. P. (2001) Intracellular delivery of a Tat-eGFP fusion protein into muscle cells. Mol. Ther. 3, 310-8.

[4] Torchilin, V. P., Levchenko, T. S., Rammohan, R., Volodina, N., PapahadjopoulosSternberg, B., and D’Souza, G. G. (2003) Cell transfection in vitro and in vivo with 
nontoxic TAT peptide- liposome-DNA complexes. Proc. Natl. Acad. Sci. U.S.A. 100, 1972- 7.

[5] Zhao, M., Kircher, M. F., Josephson, L., and Weissleder, R. (2002) Differential conjugation of tat peptide to superparamagnetic nano- particles and its effect on cellular uptake. Bioconjugate Chem. 13, 840-4.

[6] Mai, J. C., Shen, H., Watkins, S. C., Cheng, T., and Robbins, P. D. (2002) Efficiency of protein transduction is cell type-dependent and is enhanced by dextran sulfate. J. Biol. Chem. 277, 30208-18.

[7] Sawant, R. M. Hurley, J. P. Salmaso, S. Kale, A. Tolcheva, E. Levchenko, T. S. and Torchilin V. P. (2006). "SMART” Drug Delivery Systems: Double-Targeted pH-Responsive Pharmaceutical Nanocarriers. Bioconjugate Chem. 17, No. 4, 943949

[8] L. Illum, (2007). Nanoparticulate systems for nasal delivery of drugs: a real improvement over simple systems? J. Pharm. Sci. 96 473-483.

[9] Coester, C., Kreuter, J., von Briesen, H., Langer. K. (2000) Preparation of avidin-labelled gelatin nanoparticles as carriers for biotinylated peptide nucleic acid (PNA). International journal of pharmaceutics 196:147-149

[10] Zwiorek, K., Kloeckner, J., Wagner, K,. Coeste, C. (2004) Gelatin nanoparticles as a new and simple gene delivery system. Journal of pharmacy and pharmaceutical sciences 7(4):2228

[11] Xu, X., Capito, R.M.,Spector, M. (2008) Delivery of plasmid IGF-1 to chondrocytes via cationizedgelatin nanoparticles. Journal of Biomedical material research 84(1): 73-83

[12] Mrksich, M., and Whitesides, G.M., Using self-assembled monolayers to understand the interactions of man-made surfaces with proteins and cells. Annu Rev Biophys Biomol Struct 1996,25,55-78.

[13] Ratner, B.D., and Bryant, S.J., Biomaterials: where we have been and where we are going. Annu Rev Biomed Eng 2004,6,41-75. 
[14] Hancock, W.O., and Howard, J. 2002. Kinesin: Processivity and chemomechanical coupling. In Molecular Motors. M. Schliwa, editor. Wiley-VCH, Weinheim, Germany.

[15] Howard, J., Hunt, A.J., and Baek, S., Assay of microtubule movement driven by single kinesin molecules. Methods Cell Biol 1993,39,137-47.

[16] Limberis, L., and Stewart, R.J., Toward kinesin-powered microdevices. Nanotechnology 2000,11,47-51.

[17]. Huang, Y.M., Uppalapati, M., Hancock, W.O., and Jackson, T.N., Microfabricated capped channels for biomolecular motor-based transport. IEEE Advanced Packaging 2005, November,(In Press).

[18] Udabage, P., McKinnon, I.R., and Augustin, M.A., The use of sedimentation field flow fractionation and photon correlation spectroscopy in the characterization of casein micelles. J Dairy Res 2003,70,453-9.

[19] Waugh, D.F. 1971. Milk proteins: chemistry and molecular biology. Academic Press, New York. 552 pp.

[20] Howard, J., Hunt, A.J., and Baek, S., Assay of microtubule movement driven by single kinesin molecules. Methods Cell Biol 1993,39,137-47.

[21] Bhattacharyya, J., and Das, K.P., Molecular chaperone-like properties of an unfolded protein, alpha(s)-casein. J Biol Chem 1999,274,15505-9.

[22] Hua, W., Young, E.C., Fleming, M.L., and Gelles, J., Coupling of kinesin steps to ATP hydrolysis. Nature 1997,388,390-3.

[23] Gelles, J., Berliner, E., Young, E.C., Mahtani, H.K., Perez-Ramirez, B., and Anderson, K., Structural and functional features of one- and two-headed biotinated kinesin derivatives. Biophys J 1995,68,276S-281S; discussion 282S.

[24]. Berliner, E., Young, E.C., Anderson, K., Mahtani, H.K., and Gelles, J., Failure of a single-headed kinesin to track parallel to microtubule protofilaments. Nature 1995,373,718-

[25] Hua, W., Young, E.C., Fleming, M.L., and Gelles, J., Coupling of kinesin steps to ATP hydrolysis. Nature 1997,388,390-3. 
[26] Cheng, L.J., Kao, M.T., Meyhöfer, E., and Guo, J., Highly Efficient Guiding of Microtubule Transport with Imprinted CYTOP Nanotracks. Small 2005,1,409-414.

[27] Lakamper, S., Kallipolitou, A., Woehlke, G., Schliwa, M., and Meyhofer, E., Single Fungal Kinesin Motor Molecules Move Processively along Microtubules. Biophys J $2003,84,1833-43$.

[28]. Block, S.M., Goldstein, L.S., and Schnapp, B.J., Bead movement by single kinesin molecules studied with optical tweezers. Nature 1990,348,348-52.

[29] Hancock, W.O., and Howard, J., Kinesin's processivity results from mechanical and chemical coordination between the ATP hydrolysis cycles of the two motor domains. Proc Natl Acad Sci U S A 1999,96,13147-52.

[30]. Turner, D.C., Chang, C., Fang, K., Brandow, S.L., and Murphy, D.B., Selective adhesion of functional microtubules to patterned silane surfaces. Biophys J 1995,69,2782-9.

[31]. Mallik, R., Carter, B.C., Lex, S.A., King, S.J., and Gross, S.P., Cytoplasmic dynein functions as a gear in response to load. Nature 2004,427,649-52.

[32] . Brouhard, G.J., and Hunt, A.J., Microtubule movements on the arms of mitotic chromosomes: Polar ejection forces quantified in vitro. Proc Natl Acad Sci U S A 2005,102,13903-13908.

[33]. Desai, A., Verma, S., Mitchison, T.J., and Walczak, C.E., Kin I kinesins are microtubule destabilizing enzymes. Cell 1999,96,69-78.

[34]. Desai, A., and Walczak, C.E., Assays for microtubule-destabilizing kinesins. Methods Mol Biol 2001,164,109-21.

[35]. Hancock, W.O. Unpublished observations.

[36]. Yokokawa, R., Yoshida, Y., Takeuchi, S., Kon, T., Sutoh, K., and Fujita, H., Evaluation of Cryopreserved Microtubules Immobilized in Microfluidic Channels for Bead-AssayBased Transportation System. IEEE Advanced Packaging 2005.

[37]. McDonald, J.C., Duffy, D.C., Anderson, J.R., Chiu, D.T., Wu, H., Schueller, O.J., and Whitesides, G.M., Fabrication of microfluidic systems in poly(dimethylsiloxane). 
Electrophoresis 2000,21,27-40.

[38]. Kim, T.S., Nanjundaswamy, H.K., Lin, C.-T., Lakamper, S., Cheng, L.J., Hoff, D., Hasselbrink, E.F., Guo, L.J., Kurabayashi, K., Hunt, A.J., and Meyhofer, E. 2003.

Biomolecular motors as novel prime movers for microTAS: microfabrication and materials issues. In 7th Int. Conf. on Micro Total Analysis Systems. Vol. 2. M.A. Northrup, K.F. Jensen, and D.J. Harrison, editors. Transducers Research Foundation, Squaw Valley, CA. $33-6$.

[39] Brunner, C., Ernst, K.H., Hess, H., and Vogel, V., Lifetime of biomolecules in polymerbased hybrid nanodevices. Nanotechnology 2004,15, S540-S548.

[40]. Verma, V., Hancock, W.O., and Catchmark, J.M., Micro- and Nanofabrication Processes for Hybrid Synthetic and Biological System Fabrication. IEEE Advanced Packaging 2005.

[41]. Uppalapati, M., Huang, Y.M., Jackson, T.N., and Hancock, W.O. Manuscript in preparation. [42]. Han, F., Wang, Y., Sims, C.E., Bachman, M., Chang, R., Li, G.P., and Allbritton, N.L., Fast electrical lysis of cells for capillary electrophoresis. Anal Chem 2003,75,3688-96.

[43]. Irimia, D., Tompkins, R.G., and Toner, M., Single-cell chemical lysis in picoliter-scale closed volumes using a microfabricated device. Anal Chem 2004,76,6137-43.

Keywords: Protein nanotechnology, 3D structure of protein, biomolecular motors, microtubules, immobilization, system design, protein stability, nanobio based diagnostic 